\title{
Lewis acid / Base-free Strategy for the Synthesis of 2-Arylthio and Selenyl Benzothiazole / Thiazole and Imidazole
}

https://doi.org/10.1515/hc-2020-0119

Received January 27, 2020; accepted January 27, 2021.

\begin{abstract}
A $\mathrm{Cu}(\mathrm{II})$-catalyzed $\mathrm{Csp}^{2}$-Se and Csp2-Sulfur bond formation was achieved with moderate to good yields without the aid of Lewis acid and base. The reaction is compatible with a wide range of heterocycles such as benzothiazole, thiazole, and imidazole. Also, this typical protocol is found to be active in thio-selenation via S-H activation. Additionally, we proposed a plausible mechanistic pathway involving $\mathrm{Cu}(\mathrm{III})$ putative intermediate.
\end{abstract}

Keywords: Selenation, thiolation, diphenyl diselenide, thioaryl and selenoaryl derivatives, chalcogenide

\section{Introduction}

Organoselenium scaffolds engrossed much attention from chemists owing to their varied pharmaceutical activity such as antioxidants [1-5], anti-inflammatory [6-8], and antimicrobial agents [9]. Additionally, the organoselenium molecular entities were assessed for anticancer [10-11], neuroprotective [12], and antiviral properties [13-14]. Recently, selenium-containing molecules have gained a great deal of significance due to the optical activities of organic materials [15-16].

The functionalization of $\mathrm{C}-\mathrm{H}$ bonds adjacent to a hetero-atom utilizing cross-coupling is a conceptually

*Corresponding author: Gullapalli Kumaraswamy, Organic Synthesis \&Process Chemistry Division, CSIR - Indian Institute of Chemical Technology, Hyderabad - 500 007, India; Academy of Scientific and Innovative Research (AcSIR), 2 Rafi Marg, New Delhi - 110 001, India; E-mail: gkswamy_iict@yahoo.co.in Guniganti Balakishan and Vykunthapu Narayanarao, Organic Synthesis \&Process Chemistry Division, CSIR - Indian Institute of Chemical Technology, Hyderabad - 500 007, India

Pagilla Shankaraiah, Deparment of Chemistry, Osmania University, Hyderabad-500 007, India ideal process. Also, this strategy represents an extremely attractive and competent route for the substitution of $\mathrm{C}-\mathrm{H}$ bond. Moreover, the strategy is considered to be straightforward and has a step-economic advantage than traditional reactions [17-18]. Primarily, this process was induced by a sub-stoichiometric transition metal in combination with additives and bases. This regioselective oxidative coupling strategy enabled the synthesis of various pharmaceutically active heterocyclic molecular motifs [19-21]. Various groups have reported the crosscoupling of $\mathrm{C}-\mathrm{H}$ bonds adjacent to a nitrogen atom to give new $\mathrm{C}-\mathrm{C}, \mathrm{C}-\mathrm{X}(\mathrm{X}=\mathrm{S}$, Se, and Te) bond containing molecules. In particular, the construction of new $\mathrm{C}-\mathrm{S}$ and $\mathrm{C}$-Se bonds via oxidative functionalization of a C(sp2)-H bond of benzothiazoles, thiazoles, benzoxazoles, azoles, benzimidazole, imidazole, and oxadiazole has been reported elegantly [22-29]. Amongst such strategies, a Lewis acid-catalyzed, stoichiometric $\mathrm{Cu}(\mathrm{II})$-mediated thiolation reaction between heteroarenes and thiols strategy offers an appealing alternative to the conventional methods [30] (Scheme 1).

However, all these methods require stoichiometric amounts of external oxidants and bases. Hence, there is still a great need for the development of sole oxidant as air and base free protocol to produce various thioaryl and selenoaryl derivatives of benzothiazoles, thiazoles, azoles, benzimidazole, and imidazole.

\section{Results and discussion}

In our continuous interest in developing base-free $\mathrm{Cu}$ catalyzed reactions [31-34], we assessed the direct selenation and thiolation of benzothiazole. Initially, conducting the reaction between benzothiazole 1a (1 equiv.) and diphenyl diselenide $\mathbf{2 a}$ (1.2 equiv.) in the presence of $\mathrm{Cu}(\mathrm{OAc})_{2}$ (100 mol\%) and heating $100{ }^{\circ} \mathrm{C}$ in 1,4-dioxane resulted in the desired product 3a at a 54\% yield (Table 1, Entry 1). When the catalyst loading reduced to $50 \mathrm{~mol} \%$, the same 


\section{Previous work:}
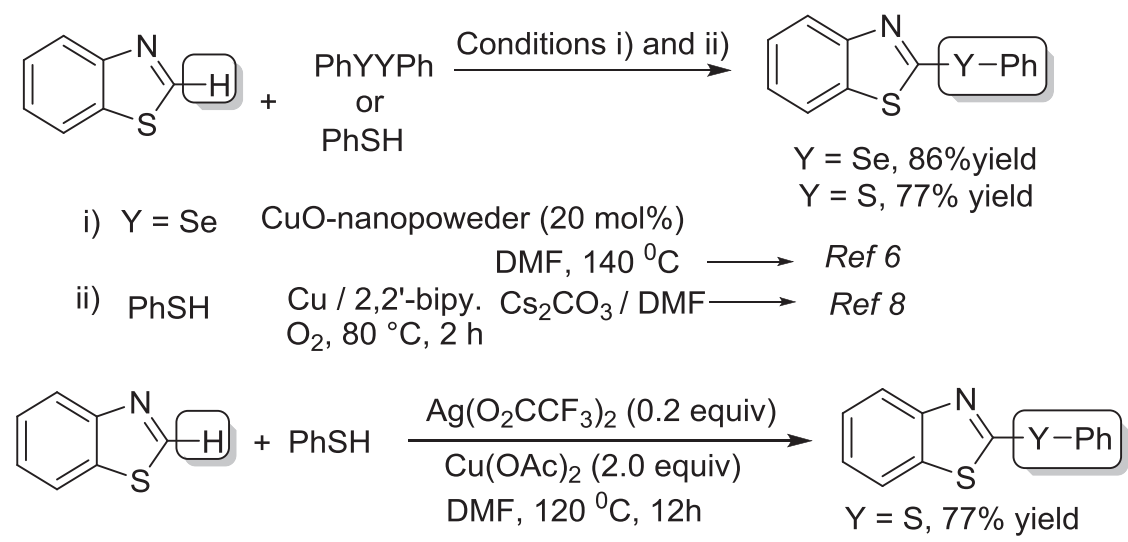

This work:

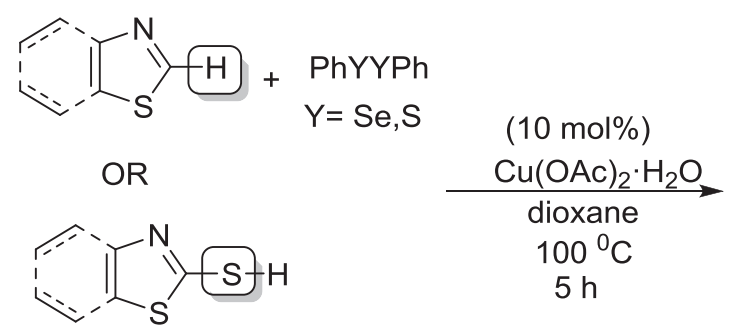

Ref 14
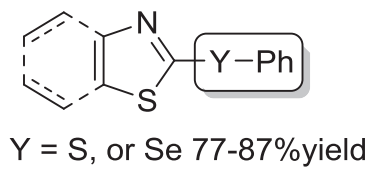

OR

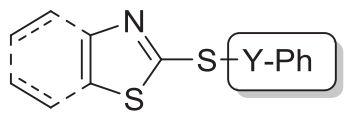

$\mathrm{Y}=\mathrm{Se} 77-87 \%$ yield

Scheme 1 Previous routs for selenation and thiolation of $\mathrm{C}-\mathrm{H}$ bonds adjacent to a hetero-atom

reaction under otherwise identical condition gave the product $3 a$ in $70 \%$ yield (Table 1, Entry 2 ).

In another reaction, the increased yield of $\mathbf{3 a}$ was observed with $20 \mathrm{~mol} \%$ of $\mathrm{Cu}$ (OAc) 2 under the same set of conditions (Table 1, Entry 3). A reaction with $10 \% \mathrm{Cu}(\mathrm{OAc}) 2$ loading under typical conditions afforded $\mathbf{3 a}$ in a decreased yield (Table 1, Entry 4). Interestingly, it was observed that the diphenyl diselenide $\mathbf{2 a}$ was not completely consumed (vide-supra) and the unreacted $\mathbf{2 a}$ was recovered at a yield of $40 \%$. When $2 \mathbf{a}$ molar ratio was reduced from $1.0 \mathrm{mmol}$ to $0.6 \mathrm{mmol}$ and carried out under identical conditions as above, this resulted in 3a with similar yields (Table 1, Entry 5). This result indicates that the by-product $\mathrm{PhSeH}$ re-oxidized to $\mathrm{PhSeSePh}$ under the atmosphere of air. Hence, it needs in an only half-molar equivalent. After considerable experimentation, we established that benzothiazole 1a (1 equiv.), diphenyl diselenide $\mathbf{2 a}$ (0.6 equiv.) and $20 \mathrm{~mol} \%$ of $\mathrm{Cu}(\mathrm{OAc}) 2$ in 1,4-dioxane at $100{ }^{\circ} \mathrm{C}$ for $5 \mathrm{~h}$ was most effective set of conditions, yielding the $\mathbf{3 a}$ at yield of $86 \%$ (Table 1, Entry 5).

Among the solvents tested, only 1,4-dioxane gave a better yield of the desired compound 3a while other solvents, such as $\mathrm{ClCH}_{2} \mathrm{CH}_{2} \mathrm{Cl}, \mathrm{CH}_{3} \mathrm{CN}$, DMF, DMSO, and $\mathrm{H}_{2} \mathrm{O}$ gave a lower yield of $\mathbf{3 a}$ (Table 1, Entries 6 to 10). Interestingly, the reaction proceeded without solvent but with albeit inferior yield (Table 1, Entry 11). A screening of copper salts indicated that $\mathrm{Cu}(\mathrm{OAc})_{2} \cdot \mathrm{H}_{2} \mathrm{O}$ performed with good efficiency for this transformation, while $\mathrm{CuI}$ and CuOTf, $\mathrm{CuCl}_{2}$, and $\mathrm{Cu}\left(\mathrm{CH}_{3} \mathrm{CN}\right)_{4} \mathrm{PF}_{6}$ led to inferior yields of 3a (Table 1, Entries 11 to 15). The $\mathrm{Co}(\mathrm{OAc})_{2}$ and $\mathrm{Pd}(\mathrm{OAc})_{2}$ as catalyst failed to initiate the reaction (Table 1, Entries 16-17). The reaction performed under an air balloon and oxygen balloon gave $\mathbf{3 a}$ in trace amount. To demonstrate the viability of this protocol, a scale-up reaction at $10 \mathrm{mmol}$ levels had also been conducted and the desired product 3a was obtained with a similar yield.

Furthermore, the reaction scope was extended by employing typical conditions and the results are shown in Table 2. To this end, $N$-methyl benzimidazole $\mathbf{1 b}$ and $\mathrm{N}$-ethyl benzimidazole $\mathbf{1 c}$ reacted in the presence of $\mathbf{2 a}$ using similar conditions. The expected products $\mathbf{3 b}$ and $\mathbf{3 c}$ were isolated in $87 \%$ and $82 \%$ yields, respectively (Table 2 , Entry 1). Likewise, $\mathrm{N}$-methyl imidazole 1d and $\mathrm{N}$-phenyl imidazole $\mathbf{1 e}$ reacted smoothly in the presence of $\mathbf{2} \mathbf{a}$ under standard protocol and the corresponding products $\mathbf{3 d}$ and 3e were isolated in $86 \%$ and $85 \%$ yields, correspondingly (Table 2, Entry 2). Remarkably, under optimized conditions, the substrates 4-methyl thiazole 1f and 5-methyl4-vinylthiazole $\mathbf{1 g}$ showed good reactivity with $\mathbf{2 a}$ in the presence of $\mathrm{Cu}(\mathrm{AcO})_{2}$ and provided the seleno derivatives 
Table 1 Optimization of reaction conditions for direct selenation ${ }^{\mathrm{a}}$

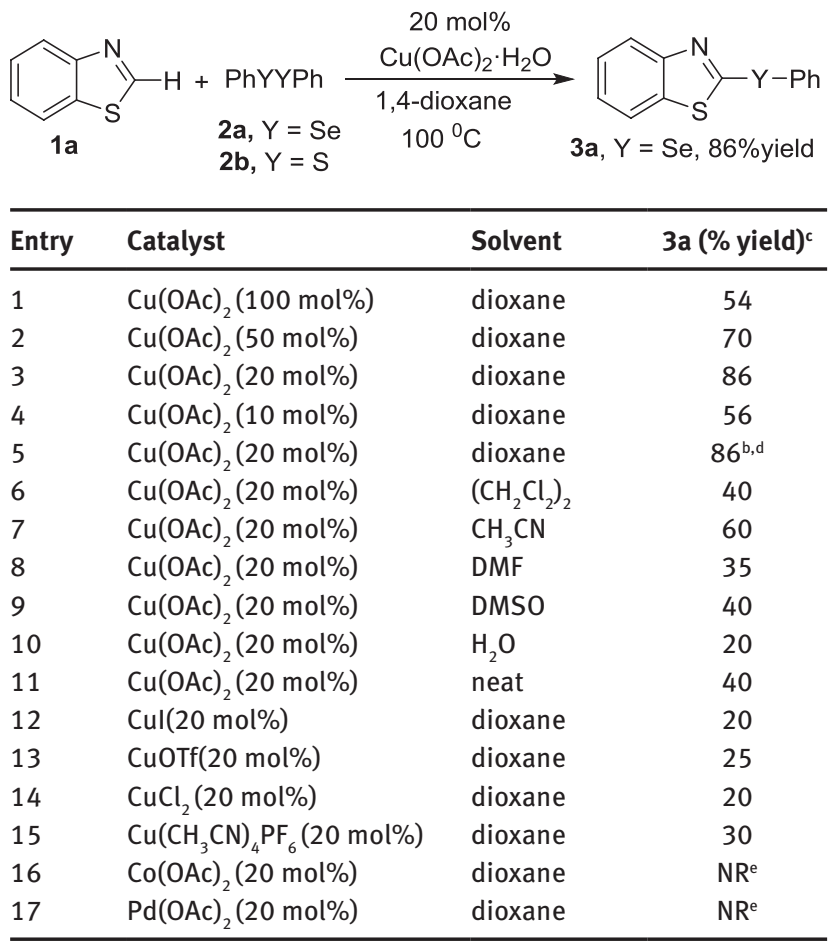

a) All reactions were carried out unless otherwise stated on the $1 \mathrm{mmol}$ scale with 1.0 equiv. $1 \mathrm{a}$ and 1.1 equiv. of $2 \mathrm{a}$ in $2 \mathrm{~mL}$ of dioxane heating at $100^{\circ} \mathrm{C}$ for $5 \mathrm{~h}$ in the open air.

b) The reaction was carried out with 1.0 equiv. $1 \mathrm{a}$ and 0.6 equiv. of $2 \mathrm{a}$. c) Isolated yield but not optimized. Yields based on the disappearance of $2 \mathrm{a}$.

d) This reaction was also carried out at $10 \mathrm{mmol}$ scale.

e) $N R=$ No reaction.

3f and 3g, in the same way (Table 2, Entries 3 and 4). The significant aspect of the reaction under this oxidative condition is that it sustains a vinyl functionality (Table 2, Entry 4). Furthermore, the direct thiolation protocol was also achieved using typical selenation conditions. Then, benzothiazole 1a, $N$-methyl benzimidazole 1b, 4-methyl thiazole $\mathbf{1 h}$ and 2-phenyl-1, 3, 4-oxadiazole were subjected to direct thiolation protocol using $\mathbf{2 b}$. As expected, the thiolation products $\mathbf{3 h}-\mathbf{k}$ were furnished in good to high yields demonstrating the generality of this reaction (Table 2, Entries 5-8).

Next, we intended to explore Cu-catalyzed S-H activation and trap the resulting organo-copper intermediate with diphenyl diselenide $\mathbf{2 a}$ under established protocol as above. Under the typical procedure, the substrates 4a-c were submitted for S-H activation. To our delight, all the substrates underwent reaction and the expected 2-phenylselenothio derivatives $\mathbf{5 a - c}$ were isolated at a yield of $80 \%$, $70 \%$, and $80 \%$, respectively (Table 3, Entries 1-3). Interestingly, this method represents a novel and mild method for the preparation of organo-sulfur-selenium containing heterocycles.

In light of this work, we now propose the following mechanism (Scheme 2). At first, copper insertion leads to the active intermediate $\mathbf{A}$ which activates benzothiazole resulting in a Cu-thiolate complex and concomitant abstraction of a $\mathrm{C}-\mathrm{H}$ by releasing $\mathrm{AcOH}$ and the copper (II) complex B. The copper (II) complex of $\mathbf{B}$ undergoes oxidation via disproportion to form $\mathrm{Cu}$ (III)-complex $\mathbf{C}$ [35]. Then, reductive elimination of $\mathrm{Cu}(\mathrm{I}) \mathrm{OAc}$ liberates the desired product. The $\mathrm{Cu}(\mathrm{I}) \mathrm{OAc}$ undergoes further oxidation in the presence of air and $\mathrm{AcOH}$ to form copper(II) acetate. A similar mechanistic pathway can be expected to perform in the synthesis of sulfur-selenium heterocycles of 5a-c. This hypothesis needs further study.

\section{Conclusion}

In conclusion, this study may contribute to a refinement of the cross-coupling of $\mathrm{C}-\mathrm{H}$ bonds, in particular, adjacent to a nitrogen atom to give new $\mathrm{C}-\mathrm{X}(\mathrm{X}=\mathrm{S}$ and $\mathrm{Se})$ bond containing molecules. Furthermore, the significant practical advantage is circumventing the stoichiometric use of base and oxidant. This protocol provides an alternative expedient synthesis of chalcogenide containing heterocyclic bioactive molecules. In particular, the synthesis of sulfur-selenium heterocycles via oxidative copper catalysis is the first of its kind to the best of our knowledge. Further work is under progress towards this end in our laboratory.

\section{Experimental Section}

All reactions were conducted under an open-air atmosphere. Apparatus used for reactions are oven-dried. 1,4-dioxane and other solvents were used as received. ${ }^{1} \mathrm{H}-\mathrm{NMR}$ spectra were recorded at 300, 400 and $500 \mathrm{MHz}$ and ${ }^{13} \mathrm{C}-\mathrm{NMR}$ at 75 and $125 \mathrm{MHz}$ in $\mathrm{CDCl}_{3} . J$ values were recorded in hertz and abbreviations used were $\mathrm{s}=$ singlet, $\mathrm{d}=$ doublet, $\mathrm{m}=$ multiplet, $\mathrm{br}=$ broad, $\mathrm{dd}=$ doublet of doublet. Chemical shifts $(\delta)$ are reported relative to TMS $(\delta=0.0)$ as an internal standard. IR (FT-IR) spectra were measured as $\mathrm{KBr}$ pellets or as films. Mass spectral data were compiled using MS (ESI), HR-MS mass spectrometers. Column chromatography was carried out using Silica gel 100-200 mesh (commercial suppliers).

A typical procedure for the Synthesis of 2-phenylseleno benzothiozole (3a): Benzothizole 1a $(1.0 \mathrm{mmol})$, diphenyl diselinide $(0.6 \mathrm{mmol})$, and $\mathrm{Cu}(\mathrm{OAc})_{2} \cdot \mathrm{H}_{2} \mathrm{O}(0.2 \mathrm{mmol}$, 
Table 2 Copper catalyzed synthesis of selenium and sulphur containing heterocycles

\begin{tabular}{|c|c|c|c|c|c|}
\hline Entry & Substrate & Product ${ }^{a, b, c}(\%$ Yield $)$ & Entry & Substrate & Product $^{\mathrm{a}, \mathrm{b}, \mathrm{c}}(\%$ Yield $)$ \\
\hline 1 & $\begin{array}{r}R \\
1 b, R=M e \\
1 c, R=E t\end{array}$ & $\begin{array}{c}\mathrm{R} \\
3 \mathrm{~b}, \mathrm{R}=\mathrm{Me}(87 \%) \\
3 \mathbf{c}, \mathrm{R}=\mathrm{Et}(82 \%)\end{array}$ & 5 & $1 a$ & $3 \mathrm{~h}(77 \%)$ \\
\hline 2 & $\begin{array}{c}R \\
1 \mathrm{~d}, \mathrm{R}=\mathrm{Me} \\
1 \mathrm{e}, \mathrm{R}=\mathrm{Ph}\end{array}$ & $\begin{array}{c}R \\
3 d, R=M e(86 \%) \\
3 e, R=P h(85 \%)\end{array}$ & 6 & 1b & $\begin{array}{c}\mathrm{Me} \\
3 \mathbf{i}(77 \%)\end{array}$ \\
\hline 3 & 1f & $3 f(85 \%)$ & 7 & $1 \mathrm{~h}$ & $\begin{array}{l}N \\
3 \mathbf{j}(78 \%)\end{array}$ \\
\hline 4 & $1 \mathrm{~g}$ & $3 \mathrm{~g}(80 \%)$ & 8 & $\mathrm{~N}-\mathrm{N}$ & $3 k(80 \%)$ \\
\hline
\end{tabular}

a) All reactions were carried out unless otherwise stated on the $1 \mathrm{mmol}$ scale with 1.0 equiv. $1 \mathrm{a}-\mathbf{i}$ and 0.6 equiv. of $2 \mathrm{a}$ and $2 \mathrm{~b}$ in $2 \mathrm{~mL}$ of dioxane heating at $100^{\circ} \mathrm{C}$ for $5 \mathrm{~h}$ in the open air.

b) Isolated yield but not optimized. Yields based on the disappearance of $\mathbf{2 a}$ and $\mathbf{2} \mathbf{b}$.

c) All products fully characterized by ${ }^{1} \mathrm{H}-\mathrm{NMR},{ }^{13} \mathrm{C}-\mathrm{NMR}$, IR, and Mass.

$20 \mathrm{~mol} \%$ ) were charged sequentially into a $10 \mathrm{~mL}$ round-bottomed flask. To this, 1,4-dioxane ( $5 \mathrm{~mL}$ ) was added and the resulting reaction mixture was stirred at $100{ }^{\circ} \mathrm{C}$ for $5 \mathrm{~h}$. Then, cooled to ambient temperature and the solvent was evaporated to give a residue that was purified over silica gel column chromatography eluting with hexane / EtOAc (9:1) to give the desired product $3 \mathrm{a}$ (185 mg, 86\%) as a yellow solid, $\mathrm{mp}$. 38-40 ${ }^{\circ} \mathrm{C}$, lit. [22] 35-36 ${ }^{\circ} \mathrm{C} .{ }^{1} \mathrm{H}-\mathrm{NMR}\left(500 \mathrm{MHz}, \mathrm{CDCl}_{3}\right): \delta 7.90$ $(\mathrm{d}, 1 \mathrm{H}, J=8.2 \mathrm{~Hz}), 7.80(\mathrm{~d}, 2 \mathrm{H}, J=8.0 \mathrm{~Hz}), 7.70(\mathrm{~d}, 1 \mathrm{H}, J=$ 7.6 Hz), 7.40-7.50 (m, 4H), 7.26-7.29 (m, 1H). ${ }^{13} \mathrm{C}-\mathrm{NMR}(75 \mathrm{MHz}$, $\left.\mathrm{CDCl}_{3}\right): \delta 162.7,154.5,136.6,130.0,129.9,126.5,125.9,124.3$, 121.9, 120.7. IR (KBr): 3056, 2969, 2923, 2851, 1739, 1453, 1418, 1368, 1307, 1067, 1018, 968, 851, 737, $686 \mathrm{~cm}^{-1}$. MS (ESI): $291(\mathrm{M}+\mathrm{H})$. HR-MS $(\mathrm{m} / \mathrm{z})$ : Calculated for $\mathrm{C}_{19} \mathrm{H}_{19} \mathrm{NSSe}$ $(M+H)=291.9684$. Found $(M+H)=291.9693$.

All other compounds including the sulfur-selenium heterocycles were synthesized $1 \mathrm{mmol}$ scale employing above typical procedure.

1-Methyl-2-(phenylselenyl)-1H-benzo(d)imidazole (3b) (Table 2, Entry 1): Yellow solid, yield $190 \mathrm{mg}$ (87\%), mp. 58-62 ${ }^{\circ} \mathrm{C} .{ }^{1} \mathrm{H}-\mathrm{NMR}\left(500 \mathrm{MHz}, \mathrm{CDCl}_{3}\right): \delta 7.80(\mathrm{~d}, 1 \mathrm{H}, J=$
8.2 Hz), 7.50-7.52 (m, 2H), 7.20-7.30 (m, 6H), 3.70 (s, 3H). ${ }^{13} \mathrm{C}-\mathrm{NMR}\left(75 \mathrm{MHz}, \mathrm{CDCl}_{3}\right): \delta 143.6,142.7,136.0,132.4,129.6$, 128.0, 123.4, 124.3, 122.5, 119.4, 109.5, 31.8. IR (KBr): 3019, 1710, 1661, 1628, 1549, 1515, 1214, 742, 667, $627 \mathrm{~cm}^{-1}$. MS (ESI): $291(\mathrm{M}+\mathrm{H})$. HR-MS: Calculated for $\mathrm{C}_{14} \mathrm{H}_{12} \mathrm{~N}_{2} \mathrm{Se}(\mathrm{M}+\mathrm{H})$ $=289.0238$. Found $(M+H)=289.0231$.

Ethyl-2-(phenylselenyl)-1H-benzo(d)imidazole (3c) (Table 2, Entry 1): Dense liquid, yield $170 \mathrm{mg}$ (82\%). ${ }^{1} \mathrm{H}-\mathrm{NMR}\left(500 \mathrm{MHz}, \mathrm{CDCl}_{3}\right): \delta$ 7.80-7.84 (m, 1H), 7.50-7.56 (m, 2H), 7.20-7.31 (m, 6H), 4.32 (q, J = 7.3 Hz, 2H), 1.24 $(\mathrm{t}, J=7.3 \mathrm{~Hz}, 3 \mathrm{H}) .{ }^{13} \mathrm{C}-\mathrm{NMR}\left(75 \mathrm{MHz}, \mathrm{CDCl}_{3}\right): \delta \quad 143.6,142.9$, 135.1, 132.4, 129.6, 128.0, 123.2, 118.9.109.6, 40.6, 14.8. IR (neat): 3055, 2977, 2927, 2852, 1609, 1576, 1414, 1343, 1251, 1152, 1102, 1067, 1020, 772, 737, 688, $668 \mathrm{~cm}^{-1}$. MS (ESI): $303(\mathrm{M}+\mathrm{H})$. HR-MS: Calculated for $\mathrm{C}_{15} \mathrm{H}_{14} \mathrm{~N}_{2} \mathrm{Se}(\mathrm{M}+\mathrm{H})=$ 303.0395. Found $=303.0387$.

1-Methyl-2-(Phenylselenyl)-1H-imidazole (3d) (Table 2, Entry 2): Yellow liquid, yield $250 \mathrm{mg}$ (86\%). ${ }^{1} \mathrm{H}-\mathrm{NMR}\left(500 \mathrm{MHz}, \mathrm{CDCl}_{3}\right): \delta$ 7.31-7.00 (m, 7H), 3.60 (s, 3H). ${ }^{13} \mathrm{C}-\mathrm{NMR}\left(75 \mathrm{MHz}, \mathrm{CDCl}_{3}\right): \delta 133.9,130.5,130.1,129.3,126.9$, 123.7, 34.7. IR (neat): 2922, 2851, 1576, 1476, 1451, 1441, 1408, 
1277, 1219, 1113, 1067, 1021, 913, 772, $687 \mathrm{~cm}^{-1}$. MS (ESI): 239 $(\mathrm{M}+\mathrm{H})$. HR-MS: Calculated for $\mathrm{C}_{10} \mathrm{H}_{10} \mathrm{~N}_{2} \mathrm{Se}(\mathrm{M}+\mathrm{H})=239.0082$. Found $=239.0077$.

1-Phenyl-2-(phenylselenyl)-1H-imidazole

(3e)

(Table2, Entry2): Yellowliquid, yield $180 \mathrm{mg}(86 \%) .{ }^{1} \mathrm{H}-\mathrm{NMR}$ $\left(500 \mathrm{MHz}, \mathrm{CDCl}_{3}\right): \delta 7.40-7.10(\mathrm{~m}, 12 \mathrm{H}) .{ }^{13} \mathrm{C}-\mathrm{NMR}(75 \mathrm{MHz}$, $\left.\mathrm{CDCl}_{3}\right): \delta 132.2,130.9,129.2,128.1,128.5,127.4,126.2$. IR (neat): 2921, 2852, 1730, 1597, 1498, 1458, 1422, 1300, 1219,

Table 3 Synthesis of sulphur-selenium heterocycles via oxidative copper catalysis

Entry

a) All reactions were carried out unless otherwise stated on the $1 \mathrm{mmol}$ scale with 1.0 equiv. $4 \mathrm{a}-\mathrm{c}$ and 0.6 equiv. of $2 \mathrm{a}$ in $2 \mathrm{~mL}$ of dioxane heating at $100^{\circ} \mathrm{C}$ for $5 \mathrm{~h}$ in the open air.

b) Isolated yield but not optimized. Yields based on the disappearance of $\mathbf{2 a}$.

c) All products fully characterized by ${ }^{1} \mathrm{H}-\mathrm{NMR},{ }^{13} \mathrm{C}-\mathrm{NMR}, \mathrm{IR}$, and Mass.
1078, 968, 772, $690 \mathrm{~cm}^{-1}$. MS (ESI): $301(\mathrm{M}+\mathrm{H})$. HR-MS: Calculated for $\mathrm{C}_{15} \mathrm{H}_{12} \mathrm{~N}_{2} \mathrm{Se}(\mathrm{M}+\mathrm{H})=301.9684$. Found $=301.9693$.

4-Methyl-2-(phenylselenyl)thiazole (3f) (Table 2, Entry 3): Yellow liquid, yield $220 \mathrm{mg}$ (85\%). ${ }^{1} \mathrm{H}-\mathrm{NMR}$ (500MHz, $\mathrm{CDCl}_{3}$ ): $\delta$ 7.58-7.60 (m, 2H), 7.38-7.40 (m, 2H), 7.21 $(\mathrm{s}, 1 \mathrm{H}), 6.70(\mathrm{~s}, 1 \mathrm{H}), 2.41(\mathrm{~s}, 3 \mathrm{H}) .{ }^{13} \mathrm{C}-\mathrm{NMR}\left(75 \mathrm{MHz}, \mathrm{CDCl}_{3}\right)$ : $\delta$ 156.3, 154.4 134.9, 132.0, 129.6, 129.0, 128.2, 116.6, 17.0. IR (neat): 3019, 1710, 1661, 1628, 1549, 1515, 1214, 742, 667, 627 $\mathrm{cm}^{-1}$. MS (ESI): $256(\mathrm{M}+\mathrm{H})$. HR-MS: Calculated for $\mathrm{C}_{10} \mathrm{H}_{9} \mathrm{NS}$ Se $(M+H)=256.0249$. Found $=256.0250$.

5-Methyl-2-(phenylselenyl)-4-vinylthiazole (3g) (Table 2, Entry 4): Yellow liquid, yield $180 \mathrm{mg}$ (80\%). ${ }^{1} \mathrm{H}-\mathrm{NMR}\left(500 \mathrm{MHz}, \mathrm{CDCl}_{3}\right): \delta 7.69-7.70(\mathrm{~m}, 2 \mathrm{H}), 7.31-7.40$ (m, 2H), 7.2 (s, 1H), 6.6 (q, 1H, $J=11.3 \mathrm{~Hz}), 5.1$ (q, 1H, $J=$ $17.3 \mathrm{~Hz}), 2.38$ (s, 3H). ${ }^{13} \mathrm{C}-\mathrm{NMR}\left(75 \mathrm{MHz}, \mathrm{CDCl}_{3}\right): \delta 180.0$, 136.0, 135.0, 135.0, 130.6, 130.3, 129.7, 129.2, 15.2. IR(neat): 2922, 2852, 1657, 1576, 1475, 1438, 1401, 1375, 1301, 1219, 1018, 999, 772,689 cm $\mathrm{cm}^{-1}$ MS (ESI): $282(\mathrm{M}+\mathrm{H})$. HR-MS: Calculated for $\mathrm{C}_{12} \mathrm{H}_{11} \mathrm{NSSe}(\mathrm{M}+\mathrm{H})=282.1021$. Found $=282.1017$.

2-(Phenylthio)benzo(d)thiazole (3h) (Table 2, Entry 5): Yellow liquid, Yield $140 \mathrm{mg}$ (77\%). ${ }^{1} \mathrm{H}-\mathrm{NMR}$ $\left(500 \mathrm{MHz}, \mathrm{CDCl}_{3}\right): \delta 7.28$ (t, $\left.1 \mathrm{H}, J=7.2 \mathrm{~Hz}\right), 7.55-7.40(\mathrm{~m}, 4 \mathrm{H})$, $7.66(\mathrm{~d}, 1 \mathrm{H}, J=8.3 \mathrm{~Hz}), 7.76(\mathrm{~d}, 2 \mathrm{H}, J=8.3 \mathrm{H}), 7.90(\mathrm{~d}, 1 \mathrm{H}, J=$ $8.2 \mathrm{~Hz}) .{ }^{13} \mathrm{C}-\mathrm{NMR}\left(75 \mathrm{MHz}, \mathrm{CDCl}_{3}\right): \quad \delta$ 169.4, 153.6, 135.1, 130.2, 129.7, 125.9, 124.1, 121.7, 120.6. IR(neat): 3019, 1710, 1661, 1628, 1549, 1515, 1214, 742, 667, $627 \mathrm{~cm}^{-1}$. MS (ESI): 244 $(\mathrm{M}+\mathrm{H})$. HR-MS: Calculated for $\mathrm{C}_{13} \mathrm{H}_{9} \mathrm{NS}_{2}(\mathrm{M}+\mathrm{H})=244.0249$. Found $=244.0244$.

1-Methyl-2-(phenylthio)-1H-benzo(d)imidazole (3i) (Table 2, Entry 6): Yellow liquid, yield $150 \mathrm{mg}$ (82\%). ${ }^{1} \mathrm{H}-\mathrm{NMR}\left(500 \mathrm{MHz}, \mathrm{CDCl}_{3}\right): \delta 7.70(\mathrm{~d}, 1 \mathrm{H}, J=7.6 \mathrm{~Hz}), 7.20$ 7.30 (m, 8H), 3.70 (s, 3H). ${ }^{13} \mathrm{C}-\mathrm{NMR}\left(75 \mathrm{MHz}, \mathrm{CDCl}_{3}\right): \delta$ 142.0, 132.0, 130, 129.4, 129.0, 127.6, 23.2, 122.4, 119.8, 109.0, 30.7. IR (neat): $\quad 3056,2923,2852,1741,1645,1611,1581,1441,1365$, $1277,1219,1153,1110,1023,1003,818,772,688,567 \mathrm{~cm}^{-1}$. MS

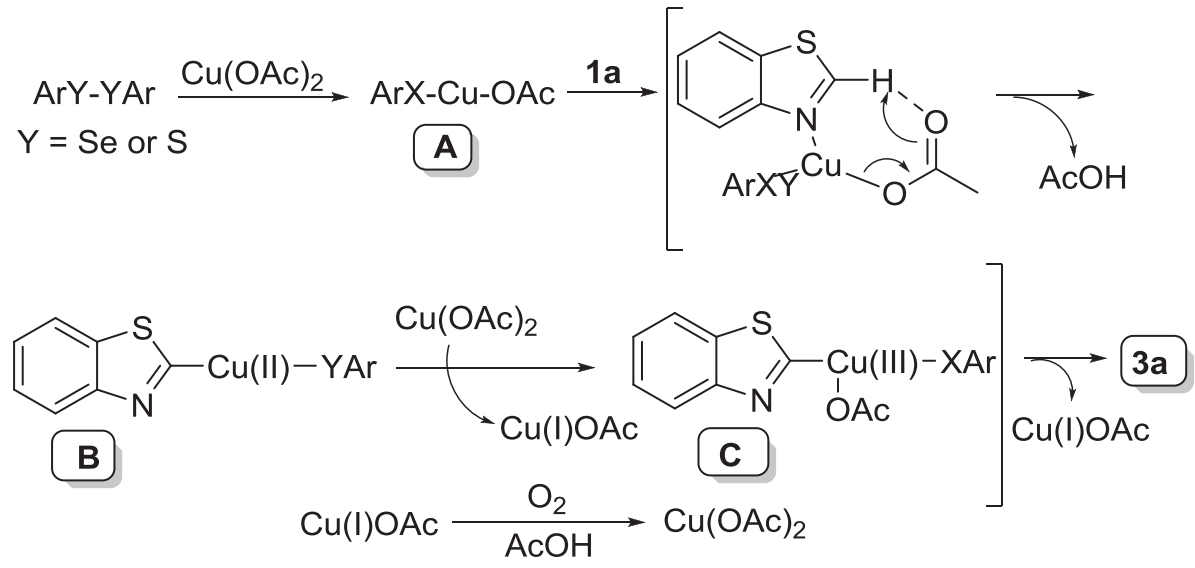

Scheme 2 Plausible catalytic cycle for the direct selenation 
(ESI): $241(\mathrm{M}+\mathrm{H})$. HR-MS: Calculated for $\mathrm{C}_{14} \mathrm{H}_{12} \mathrm{~N}_{2} \mathrm{~S}(\mathrm{M}+\mathrm{H})=$ 241.0794. Found $=241.0793$.

4-Methyl-2-(phenylthio)thiazole (3j) (Table 2, Entry 7): Yellow liquid, yield $175 \mathrm{mg}(78 \%) .{ }^{1} \mathrm{H}-\mathrm{NMR}(500 \mathrm{MHz}$, $\left.\mathrm{CDCl}_{3}\right): \delta$ 7.58-7.60 (m, 2H), 7.38-7.40 (m, 2H), 7.21 (s, $\left.1 \mathrm{H}\right)$, $6.70(\mathrm{~s}, 1 \mathrm{H}), 2.41(\mathrm{~s}, 3 \mathrm{H}) .{ }^{13} \mathrm{C}-\mathrm{NMR}\left(75 \mathrm{MHz}, \mathrm{CDCl}_{3}\right): \delta 164.7$, 153.5 133.5, 132.0, 129.6, 129.3, 115.0, 17.1. IR (neat): 3019.7, 1710, 1661.4, 1628, 1549, 1515.3, 1214.8, 742, 667, $627 \mathrm{~cm}^{-1}$. MS (ESI): $208(\mathrm{M}+\mathrm{H})$. HR-MS: Calculated for $\mathrm{C}_{10} \mathrm{H}_{9} \mathrm{NS}_{2}$ $(M+H)=208.0249$. Found $=208.0250$.

2-Phenyl-5-(phenylthio)-1, 3, 4-oxadiazole (3k) (Table 2, Entry 8): Yellow liquid, yield $140 \mathrm{mg}(80 \%)$. ${ }^{1} \mathrm{H}-\mathrm{NMR}\left(500 \mathrm{MHz}, \mathrm{CDCl}_{3}\right): \delta$ 7.97-7.90 (m, 2H), 7.69-7.60 (m, $2 \mathrm{H})$, 7.50-7.40 (m, 6H). ${ }^{13} \mathrm{C}-\mathrm{NMR}\left(75 \mathrm{MHz}, \mathrm{CDCl}_{3}\right): \delta 166.3$, 162.8, 133.6, 131.7, 129.7, 128.9, 126.7, 123.4. IR (neat): 3019, 1710, 1661, 1628, 1549, 1515, 1214, 742, 667, $627 \mathrm{~cm}^{-1}$. MS (ESI): $255(\mathrm{M}+\mathrm{H})$. HR-MS: Calculated for $\mathrm{C}_{14} \mathrm{H}_{10} \mathrm{~N}_{2} \mathrm{OS} \quad(\mathrm{M}+\mathrm{H})=$ 255.9684. Found $=255.9693$.

\section{2-((Phenylselanyl)thio)benzo(d)thiazole}

(Table 3, Entry 1): Yellow liquid, yield $155 \mathrm{mg}$ (77\%). ${ }^{1} \mathrm{H}-\mathrm{NMR}\left(500 \mathrm{MHz}, \mathrm{CDCl}_{3}\right): \delta 7.90(\mathrm{~d}, 1 \mathrm{H}, J=8.2 \mathrm{~Hz}), 7.70$ (d, $1 \mathrm{H}, J=7.6 \mathrm{~Hz}), 7.50-7.61(\mathrm{~m}, 2 \mathrm{H}), 7.24-7.28(\mathrm{~m}, 5 \mathrm{H}) .{ }^{13} \mathrm{C}-\mathrm{NMR}$ (75MHz, $\left.\mathrm{CDCl}_{3}\right): \delta 131.4,129.8,129.5,129.1,128.9,127.6,126.5$, 125.2, 122.5, 121.2. IR (neat): 2956, 2918, 2850, 1727, 1462, 1426.3, 1377.8, 1265, 1123, 1077, 1003, 972, 909, 727, $687 \mathrm{~cm}^{-1}$. MS (ESI): $323(\mathrm{M}+\mathrm{H})$. HR-MS: Calculated for $\mathrm{C}_{13} \mathrm{H}_{9} \mathrm{NSeS}_{2}$ $(\mathrm{M}+\mathrm{H})=323.9414$. Found $=323.9409$.

2-Phenyl-5-((phenylselanyl)thio)-1,3,4-oxadiazole (5b) (Table 3, Entry 2): Yellow liquid, Yield $160 \mathrm{mg}$ (70\%). ${ }^{1} \mathrm{H}-\mathrm{NMR}\left(500 \mathrm{MHz}, \mathrm{CDCl}_{3}\right): \delta 7.90(\mathrm{~d}, 1 \mathrm{H}, J=7.5 \mathrm{~Hz}), 7.20$ 7.61 (m, 9H). ${ }^{13} \mathrm{C}-\mathrm{NMR}\left(75 \mathrm{MHz}, \mathrm{CDCl}_{3}\right): \delta$ 133.2, 132.0, 131.4, 129.5, 129.1, 127.6, 126.8. IR(neat): 2919, 2851, 1781, 1726, 1609, 1578, 1576, 1549, 1465, 1349, 1286, 1219, 1170, 1066, 1023, 956, 772, $689 \mathrm{~cm}^{-1}$. MS (ESI): $335(\mathrm{M}+\mathrm{H})$. HR-MS: Calculated for $\mathrm{C}_{14} \mathrm{H}_{10} \mathrm{~N}_{2} \mathrm{OSSe}(\mathrm{M}+\mathrm{H})=335.1021$. Found $=$ 335.1017.

1-Phenyl-5-((phenylselanyl)thio)-1H-tetrazole (5c) (Table 3, Entry 3): Yellow liquid, yield $150 \mathrm{mg}(80 \%)$. ${ }^{1} \mathrm{H}-\mathrm{NMR}\left(500 \mathrm{MHz}, \mathrm{CDCl}_{3}\right): \delta 7.70$ (d, $1 \mathrm{H}, J=7.5 \mathrm{~Hz}$ ), 7.60-7.20 (m, 9H). ${ }^{13} \mathrm{C}-\mathrm{NMR}\left(75 \mathrm{MHz}, \mathrm{CDCl}_{3}\right): \delta$ 153.2, 150.9, 149.9, 149.2, 149.1, 149.0, 148.6 147.1, 144.0. IR (neat): 3019, 1710, 1661, 1628, 1549, 1515, 1214, 742, 667, $627 \mathrm{~cm}^{-1}$. MS (ESI): $334(\mathrm{M}+\mathrm{H})$. HR-MS: Calculated for $\mathrm{C}_{13} \mathrm{H}_{10} \mathrm{~N}_{4} \mathrm{SSe}(\mathrm{M}+\mathrm{H})=334.9864$; Found $(\mathrm{M}+\mathrm{H})=334.9860$.

Supporting Information: Copies of ${ }^{1} \mathrm{H}$ and ${ }^{13} \mathrm{C}$ NMR spectra are available.

Acknowledgements: ORGIN program (CSIR) and CSIR (New Delhi) is gratefully acknowledged for awarding the fellowship to G. B and VN.
Research funding: Financial support was provided by the DST, New Delhi, India (Grant No: SR/S1/OC-08/2011).

Conflict of interest: Authors state no conflict of interest.

Data availability statement: All data generated or analyzed during this study are included in this published article and its supplementary information files.

\section{References}

[1] Jain VK. Chapter 1: An Overview of Organoselenium Chemistry: From Fundamentals to Synthesis. In: Jain VK, Priyadarsini KI, editors. Organoselenium Compounds in Biology and Medicine. Synthesis, Biological and Therapeutic Treatments. Royal Chemical Society; 2017. p. 1-33.

[2] Iwaoka M. Antioxidant Organoselenium Molecules. In: Santi C, editor. Organoselenium Chemistry Between Synthesis and Biochemistry. Bentham Science Publishers; p. 361-78.

[3] Sies H. Ebselen, a selenoorganic compound as glutathione peroxidase mimic. Free Radic Biol Med. 1993 Mar;14(3):313-23.

[4] Nascimento V, Alberto EE, Tondo DW, Dambrowski D, Detty MR, Nome F, et al. GPx-Like activity of selenides and selenoxides: experimental evidence for the involvement of hydroxy perhydroxy selenane as the active species. J Am Chem Soc. 2012 Jan;134(1):138-41.

[5] Nogueira CW, Zeni G, Rocha JB. Organoselenium and organotellurium compounds: toxicology and pharmacology. Chem Rev. 2004 Dec;104(12):6255-85.

[6] Nogueira CW, Quinhones EB, Jung EA, Zeni G, Rocha JB. Anti-inflammatory and antinociceptive activity of diphenyl diselenide. Inflamm Res. 2003 Feb;52(2):56-63.

[7] Pavin NF, Donato F, Cibin FW, Jesse CR, Schneider PH, de Salles $\mathrm{HD}$, et al. Antinociceptive and anti-hypernociceptive effects of Se-phenyl thiazolidine-4-carboselenoate in mice. Eur J Pharmacol. 2011 Oct;668(1-2):169-76.

[8] Martínez-Ramos F, Salgado-Zamora H, Campos-Aldrete ME, Melendez-Camargo E, Márquez-Flores Y, Soriano-García M. Synthesis and anti-inflammatory activity evaluation of unsymmetrical selenides. Eur J Med Chem. 2008 Jul;43(7):1432-7.

[9] Sancineto L, Piccioni M, De Marco S, Pagiotti R, Nascimento V, Braga AL, et al. Diphenyl diselenide derivatives inhibit microbial biofilm formation involved in wound infection. BMC Microbiol. 2016 Sep;16(1):220-30.

[10] Álvarez-Pérez M, Ali W, Marć MA, Handzlik J, DomínguezÁlvarez E. Selenides and Diselenides: A Review of Their Anticancer and Chemopreventive Activity. Molecules. 2018 Mar;23(3):628-47.

[11] Almeida GM, Rafique J, Saba S, Siminski T, Mota NS, Filho DW, et al. Novel selenylated imidazo[1,2-a]pyridines for breast cancer chemotherapy: inhibition of cell proliferation by Akt-mediated regulation, DNA cleavage and apoptosis. Biochem Biophys Res Commun. 2018 Sep;503(3):1291-7.

[12] Pinton S, da Rocha JT, Gai BM, Prigol M, da Rosa LV, Nogueira CW. Neuroprotector effect of $p, p^{\prime}$-methoxyl-diphenyl diselenide in a model of sporadic dementia of Alzheimer's type in mice: 
contribution of antioxidant mechanism. Cell Biochem Funct. 2011 Apr;29(3):235-43.

[13] Lieberman OJ, Orr MW, Wang Y, Lee VT. High-throughput screening using the differential radial capillary action of ligand assay identifies ebselen as an inhibitor of diguanylate cyclases. ACS Chem Biol. 2014 Jan;9(1):183-92.

[14] Sartori G, Jardim NS, Marcondes Sari MH, Dobrachinski F, Pesarico AP, Rodrigues LC Jr, et al. Antiviral Action of Diphenyl Diselenide on Herpes Simplex Virus 2 Infection in Female BALB/c Mice. J Cell Biochem. 2016 Jul;117(7):1638-48.

[15] Abdallah M, Hijazi A, Graff B, Fouassier JP, Rodeghiero G, Gualandi A, et al. Coumarin derivatives as versatile photoinitiators for 3D printing, polymerization in water and photocomposite synthesis. Polym. Chem. 2019;10(7):872-84.

[16] Dayam R, Gundla R, Al-Mawsawi LQ, Neamati N. HIV-1 integrase inhibitors: 2005-2006 update. Med Res Rev. 2008 Jan;28(1):118-54.

[17] Beletskaya IP, Ananikov VP. Transition-metal-catalyzed C-S, C-Se, and C-Te bond formation via cross-coupling and atom-economic addition reactions. Chem Rev. 2011 Mar;111(3):1596-636.

[18] Guo XX, Gu DW, Wu Z, Zhang W. Copper-catalyzed C-H functionalization reactions: efficient synthesis of heterocycles. Chem Rev. 2015 Feb;115(3):1622-51.

[19] Wang Y, Chackalamannil S, Chang W, Greenlee W, Ruperto V, Duffy RA, et al. Design and synthesis of ether analogues as potent and selective M2 muscarinic receptor antagonists. Bioorg Med Chem Lett. 2001 Apr;11(7):891-4.

[20] Gangjee A, Zeng Y, Talreja T, McGuire JJ, Kisliuk RL, Queener SF. Design and synthesis of classical and nonclassical 6-arylthio2,4-diamino-5-ethylpyrrolo[2,3-d]pyrimidines as antifolates. J Med Chem. 2007 Jun;50(13):3046-53.

[21] Liu G, Link JT, Pei Z, Reilly EB, Leitza S, Nguyen B, et al. Discovery of novel p-arylthio cinnamides as antagonists of leukocyte function-associated antigen-1/intracellular adhesion molecule-1 interaction. 1. Identification of an additional binding pocket based on an anilino diaryl sulfide lead. J Med Chem. 2000 Oct;43(21):4025-40.

[22] Rosario AR, Casola KK, Oliveira CE, Zeni G. Copper oxide nanoparticle-catalyzed chalcogenation of the carbon-hydrogen bond in thiazoles: synthesis of 2-(organochalcogen)thiazoles. Adv Synth Catal. 2013;355(14-15):2960-6.

[23] Fukuzawa SI, Shimizu E, Atsuumi Y, Haga M, Ogata K. Coppercatalyzed direct thiolation of benzoxazole with diaryl disulfides and aryl thiols. Tetrahedron Lett. 2009;50(20):2374-6.
[24] Ranjit S, Lee R, Heryadi D, Shen C, Wu J, Zhang P, et al. Coppermediated C-H activation/C-S cross-coupling of heterocycles with thiols. J Org Chem. 2011 Nov;76(21):8999-9007.

[25] Murru S, Ghosh H, Sahoo SK, Patel BK. Intra- and intermolecular C-S bond formation using a single catalytic system: first direct access to arylthiobenzothiazoles. Org Lett. 2009 Oct;11(19):4254-7.

[26] Popov I, Do HQ, Daugulis O. In situ generation and trapping of aryllithium and arylpotassium species by halogen, sulfur, and carbon electrophiles. J Org Chem. 2009 Nov;74(21):8309-13.

[27] Nakamura S, Tsuno N, Yamashita M, Kawasaki I, Ohta S, Ohishi Y. Synthesis of a regio-isomer of kealiiquinone, a marine benzimidazole alkaloid. J Chem Soc, Perkin Trans 1. 2001;4(4):429-36.

[28] Sharma P, Rohilla S, Jain N. Copper acetate-DMSO promoted methylthiolation of arenes and heteroarenes. J Org Chem. 2015 Apr;80(8):4116-22.

[29] Wertz S, Kodama S, Studer A. Amination of benzoxazoles and 1,3,4-oxadiazoles using 2,2,6,6-tetramethylpiperidine- $N$ oxoammonium tetrafluoroborate as an organic oxidant. Angew Chem Int Ed Engl. 2011 Nov;50(48):11511-5.

[30] Dai C, Xu Z, Huang F, Yu Z, Gao YF. Lewis acid-catalyzed, copper(II)-mediated synthesis of heteroaryl thioethers under base-free conditions. J Org Chem. 2012 May;77(9):4414-9.

[31] Kumaraswamy G, Jayaprakash N, Balakishan G. Cu(I)-catalyzed tandem benzyldiazoester coupling with terminal alkyne-allene formation-Michael reaction: application to the syntheses of oxa and azacycles. Org Biomol Chem. 2011 Oct;9(22):7913-20.

[32] Kumaraswamy G, Balakishan G. Copper(I)-catalysed Domino coupling and cyclisation reaction: a mild, expedient toute for the synthesis of indene and dihydronaphthalene derivatives. Eur J Org Chem. 2015;2015(14):3141-6.

[33] Kumaraswamy G, Rao GV. RamaKrishna, G. Stereocontrolled copper iodide catalyzed phosphorus-carbon bond formation: an efficient synthesis of scalemic tertiary phosphineboranes. Synlett. 2006;7:1122-4.

[34] Kumaraswamy G, Rambabu D, Jayaprakash N, Venkata Rao G, Sridhar B. Cu- and Mo-catalysed expedient synthesis of alkynyl-substituted derivatives of 1,2-dihydropyridines, -quinolines and -isoquinolines. Eur J Org Chem. 2009;2009(24):4158-64.

[35] King AE, Huffman LM, Casitas A, Costas M, Ribas X, Stahl SS. Copper-catalyzed aerobic oxidative functionalization of an arene $\mathrm{C}-\mathrm{H}$ bond: evidence for an aryl-copper(III) intermediate. J Am Chem Soc. 2010 Sep;132(34):12068-73. 